\title{
An update on prophylactic human papillomavirus (HPV) vaccines: a review of key literature published between September 2018 and September 2019
}

\author{
Anja Šterbenc ${ }^{1}$, Tina Triglav ${ }^{1}$, Mario Poljak ${ }^{1 凶}$
}

\begin{abstract}
Prophylactic human papillomavirus (HPV) vaccines have revolutionarily modified our understanding of and efforts in preventing communicable diseases. Undeniably, all three prophylactic HPV vaccines currently available have excellent safety and have substantially contributed to the control of HPV infections and HPV-related diseases during the past decade in the setting where high vaccine coverage has been achieved. This review summarizes and discusses the most influential peer-reviewed literature on HPV vaccines published between September 2018 and September 2019. The review focuses on the current status of HPV vaccination implementation, results from recent clinical trials, updates to recommendations and guidelines, long-term immunogenicity, and evaluations of various dosing schemes and HPV vaccination of alternative populations. In addition, we briefly summarize studies on the real-life effectiveness of prophylactic HPV vaccines from countries with successful HPV vaccination programs, present an update on safety data, and discuss progress and the development of novel prophylactic HPV vaccines, as well as potential future applications and challenges.
\end{abstract}

Keywords: human papillomavirus, prophylactic HPV vaccines, literature review

Received: 24 September 2019| Returned for modification: 9 November 2019| Accepted: 13 November 2019

\section{Introduction}

Currently licensed and available prophylactic human papillomavirus (HPV) vaccines include a bivalent (2vHPV) vaccine against HPV16/18 (Cervarix; GlaxoSmithKline Biologicals, Belgium), a quadrivalent (4vHPV) vaccine against HPV6/11/16/18 (Gardasil; Merck \& Co., USA / Silgard; Sanofi Pasteur MSD, France), and a nonavalent (9vHPV) vaccine against HPV6/11/16/18/31/33/45/52/58 (Gardasil9; Merck \& Co., USA). All three HPV vaccines have been shown to be highly effective and extremely safe, not only in rigorous evaluations within clinical trials but also in real-world settings and post-licensure monitoring $(1,2)$.

As described in detail previously (1), before the start of the annual national HPV vaccination campaign in Slovenia, the Section for School, University, and Adolescent Health of the Slovenian Medical Society has been holding a yearly professional 1-day "refresher course" dedicated to promotion of and education on HPV vaccination. This year, we celebrated the 1oth anniversary since the introduction of free-of-charge HPV vaccination into the Slovenian immunization program, which prompted the coordinators to hold a special meeting entitled "10 years of HPV vaccination" held on September 3rd, 2019 in Ljubljana, Slovenia, with a focus on the successes of prophylactic HPV vaccines. The majority of the publications presented in this literature review were discussed at this meeting. The purpose of the review was to update and improve the knowledge of healthcare workers responsible for HPV vaccination, including school medicine specialists, pediatricians, gynecologists, epidemiologists, and others. This review summarizes the most important peer-reviewed literature published between September 2018 and September 2019, which corresponds to the 2018/2019 school year in Slovenia.

\section{Methods}

For the professional meeting and this literature review, we searched PubMed for the terms "human papillomavirus vaccine" and "HPV vaccine" with a custom date range filter of September 1st, 2018 to September 1st, 2019. The main focus of our manual screening of the 1,011 matches retrieved were publications on the HPV vaccine potential and uptake, changes to the existing recommendations and guidelines, efficacy and immunogenicity clinical trials, studies evaluating various dosing schemes and vaccination of alternative populations, studies contributing to the mounting evidence of the real-life effectiveness of prophylactic HPV vaccines even from countries with modest HPV vaccination coverage rates, updates on HPV vaccine safety data, and progress in the development of novel prophylactic HPV vaccines.

\section{HPV vaccination status at the global level}

Since 2006, prophylactic HPV vaccines have been increasingly introduced worldwide. As of early 2019, a total of 115 countries or territories have implemented HPV vaccination into their national programs, including 24 countries or territories that provide gender-neutral HPV vaccination. By the end of 2021, 39 countries or territories are additionally projected to have a national HPV vaccination program, provided that the global vaccine supply is sufficient (3).

\section{Recommendations, guidelines, and scientific advice: an update}

On October 5th, 2018 the US Food and Drug Administration (FDA) 
announced extension of the indications of the 9vHPV vaccine to include men and women age 27 through 45 . The approval was largely based on the results of a study of 3,200 women age 27 to 45 in whom the 9vHPV vaccine proved to be $88 \%$ effective in preventing persistent HPV infections, genital warts, preneoplastic lesions of the vulva, vagina, and cervix, and cervical cancer (4). After reviewing evidence on HPV vaccination in adults, the US Advisory Committee on Immunization Practices (ACIP) updated the recommendations for catch-up vaccination and vaccination of adults in June 2019. Whereas routine recommendations for adolescents have not changed since the last published recommendations in 2016, ACIP now recommends catch-up HPV vaccination for all individuals through age 26. For adults 27 to 45 years old, catch-up HPV vaccination is not routinely recommended; ACIP recommends shared clinical decision-making regarding HPV vaccination for those that were not adequately vaccinated (5). In their position paper, the European Society of Gynaecological Oncology and the European Federation for Colposcopy also strongly support gender-neutral vaccination programs for children and young adolescents, together with a catch-up program for young adults and, whenever feasible, HPV vaccination on an individual basis (6).

In April 2019, the European Centre for Disease Prevention and Control (ECDC) opened a public consultation on the new guidance for the introduction of HPV vaccines in EU countries, focusing especially on the 9vHPV vaccine, addition of boys to the girls-only HPV vaccination programs, and HPV vaccination of people living with HIV (PLWH). Since all EU/EEA member states successfully implemented HPV vaccination into their national vaccination programs and several countries also expended their recommendations to include boys, questions regarding the effectiveness and especially cost-effectiveness of this approach have been raised. The key conclusions of the draft were: i) the 4vHPV and 9vHPV vaccines are highly efficacious and immunogenic in both women and men, ii) currently there is no direct evidence of clinical efficacy of HPV vaccines in PLWH, although new evidence is emerging from ongoing studies, and iii) the results of cost-effectiveness analysis depend on the endpoint investigated; if the priority is to prevent cervical disease in women, adding boys to current national girls-only HPV immunization programs becomes increasingly more cost-effective if vaccination coverage rates among females are persistently low and if the costs of HPV vaccine are reduced. Nevertheless, increasing vaccination coverage rates among girls might still be more cost-effective; however, if the HPV vaccination program is primarily used to prevent all HPV-related disease, gender-neutral HPV vaccination could be the most cost-effective strategy (7).

\section{Clinical trials}

The prevalence of HPV, cytological abnormalities, and cervical intraepithelial neoplasia grade 2 or worse (CIN2+) were assessed using the results of the baseline phase of the BD Onclarity HPV Trial, which included 14,153 US women 21 to 34 years old undergoing routine cervical cancer screening in order to evaluate the impact of largely opportunistic HPV vaccination in the United States. Interestingly, despite lower HPV vaccine coverage rates and vaccination in patients older than the recommended routine age at first dose, there was a statistically significant decrease in the prevalence of overall HPV infections, HPV vaccine targeted types (HPV16 from 31 to $83 \%$, and HPV18 from 73 to $89 \%$ ), and even HPV types that are not covered by the HPV vaccine (HPV31 and HPV33/58) among vaccinated women regardless of the age group compared to those that did not receive the HPV vaccine. Moreover, compared to unvaccinated women, vaccinated women were less likely to have a cytology result of low-grade squamous intraepithelial lesions (LSIL) or worse and CIN2+, suggesting that "catch-up" vaccination provides benefits for adolescents and young adults (8).

In order to assess the efficacy of the 9vHPV vaccine against cervical disease and cervical surgeries related to the vaccine types, three international, randomized, double-blind trials were conducted using a historic placebo population for comparison. These studies found a 98.2\% (95\% confidence interval [CI], 93.6-99.7) and $97.8 \%$ (95\% CI, 93.4-99.4) reduction in the incidence of highgrade cervical disease and cervical surgery, respectively, related to the HPV types included in the 9vHPV vaccine. Although the 9vHPV vaccine did not prevent disease related to the vaccine HPV types that had been detected prior to vaccination, it considerably reduced the incidence of cervical, vaginal, and vulvar disease associated with other vaccine HPV types, suggesting that early vaccination in HPV-naive individuals is most effective, whereas sexually active individuals may still benefit from catch-up vaccination programs (9).

SPERANZA (SPERimentazione Anti HPV Zona Apuana) is a clinical trial designed to assess the clinical effectiveness of HPV vaccination following surgical treatment of $\mathrm{CIN} 2+$ lesions and microinvasive cervical cancer. In women that had undergone conization, the $4 \mathrm{vHPV}$ vaccine exhibited $80 \%$ clinical effectiveness in disease relapse prevention, with clinical benefits demonstrated up to 4 years post vaccination. Although the $4 \mathrm{vHPV}$ vaccine lacked a therapeutic effect on prevalent HPV infection and disease, it may be used as an adjuvant to surgical treatment of cervical disease (10).

Because more than a third of patients with high-grade squamous intraepithelial lesions (HSIL) will experience disease recurrence after initial treatment, a randomized, double-blind, placebo-controlled, proof-of concept clinical trial has been recently initiated to determine whether the 9vHPV vaccine can reduce the risk of anal and vulvar HSIL recurrence by 50\% in previously unvaccinated individuals that recently received treatment for anal or vulvar HSIL. The trial is set to include 345 eligible participants 27 to 69 years old with a history of anal or vulvar HSIL that have not yet received the HPV vaccine. The study is predicted to close in 2022 and, if successful, prophylactic vaccines may become part of the standard treatment of anal and vulvar HSIL (11).

\section{Long-term immunogenicity of HPV vaccines}

It has been firmly established in various clinical trials that prophylactic HPV vaccines induce a robust protective immune response; however, real-life data on the long-term stability of vaccine-induced antibodies and the duration of immunity conferred by HPV vaccines are less abundant.

A comparison of long-term antibody responses induced by the 2vHPV and 4vHPV vaccines was evaluated in a cohort of Finnish females that participated in the phase III HPV vaccination trials FUTURE II in 2002 and PATRICIA in 2004, with subsequent serum sample linkage to the Finnish Maternity cohort biobank (12). The study found that, for the majority of vaccinated women, anti-HPV16 and anti-HPV18 antibody levels were higher than the natural infection-related antibody levels and remained stable for 
up to 12 years post vaccination, confirming long-term protection. Interestingly, whereas the antibody levels induced by the 2vHPV vaccine were high and stable in nearly $100 \%$ of women even after 12 years, anti-HPV18 antibody levels declined below the natural infection antibody level in $18 \%$ of women that had received the $4 \mathrm{vHPV}$ vaccine, suggesting potential differences in immune responses to the two HPV vaccines (12).

To provide data on the duration of immunity induced by different HPV vaccination schedules and the role of a booster dose, a follow-up nonrandomized clinical trial was performed on Mexican girls and women vaccinated with either the $2 \mathrm{vHPV}$ or $4 \mathrm{vHPV}$ vaccine (13). The geometric mean titers (GMTs) for HPV16 and HPV 18 at 5 years post vaccination were shown to be above levels induced by natural infection, confirming non-inferiority of a twodose schedule. In addition, due to the establishment of robust immune memory, a long-lasting humoral response is induced, suggesting that boosters are unnecessary and unlikely to be profitable (13).

There is evidence that the $2 \mathrm{VHPV}$ vaccine induces cross-reactive immunogenicity against HPV31 and HPV45; however, little is known on the durability of this phenomenon. Based on recent data from a subset of subjects previously enrolled in phase I/II and phase IIIb clinical trials of the 2vHPV vaccine, it appears that cross-reactivity is long-lasting with comparable GMTs in girls 9 to 14 years old that had received two doses and women 15 to 25 years old that had received three doses for up to 5 years after the first vaccination. Moreover, specific $\mathrm{CD}_{4}+\mathrm{T}$-cell responses against HPV $_{31}$ and HPV 45 were similar across groups at month 36, with the observed presence of specific memory B cells additionally confirming establishment of cross-protection (14).

More data are becoming available regarding the efficacy of HPV vaccines in HPV-related oropharyngeal cancers. A study evaluating antibody responses in serum samples and oral gargles collected from 150 US men 27 to 45 years old that had received three doses of the $4 \mathrm{vHPV}$ vaccine has shown that all participants developed detectable anti-HPV16 and anti-HPV18 antibodies in serum at month 7 with somewhat lower but still high positivity rates in oral gargle samples (93.2\% for HPV16 and $72.1 \%$ for HPV18). However, the proportion of men with detectable oral antibodies decreased significantly at 18 and 30 months after vaccination (39.8\% and 29.6\% for anti-HPV16 antibodies, and 10.7\% and 4.6\% for anti-HPV18 antibodies, respectively). Nevertheless, when detectable, oral gargle anti-HPV antibody levels remained strongly correlated with anti-HPV serum antibody levels (15).

\section{Dosing schemes}

In 2014, the World Health Organization (WHO) changed the original three-dose HPV vaccine schedule, recommending a two-dose schedule for those starting HPV vaccination at age 14 or younger and a three-dose schedule for individuals that start vaccination at age 15 or older and those that are immunocompromised; this was mostly based on observational studies and immunogenicity trials (16). It was already previously shown that the number of vaccine doses received and the timing between them is crucial for 4vHPV vaccine effectiveness against anogenital warts $(17,18)$, whereas data on effectiveness against $\mathrm{CIN} 2+$ with respect to the age at 4VHPV vaccination and number of doses were lacking. A recent nationwide population-based study on vaccinated and unvaccinated Danish and Swedish women found a reduced risk for $\mathrm{CIN}_{2}+$ for those that had been vaccinated with three doses of
$4 \mathrm{VHPV}$ vaccine before age 20 , whereas women that had had their second dose at least 5 months after the first dose did not have an increased risk of $\mathrm{CIN} 2+$ after two doses compared to those receiving three doses (19).

The updated evidence from an Indian follow-up cohort study on the efficacy of less than three doses of the vaccine clearly shows that the protection conferred by a two-dose regimen of $4 \mathrm{VHPV}$ vaccine against incident and persistent HPV infection in girls 15 to 18 years old is comparable to that of the three-dose regimen in girls 15 to 18 years old, providing immune-bridging data to allow extension of the target age for HPV vaccination of girls up to 18 years with two doses (20).

Although all three prophylactic HPV vaccines have been in use for several years now, little is known about the use of different HPV vaccines within the same individual. Hence, a randomized clinical trial was conducted to evaluate immune response when administrating two different HPV vaccines in varying order. Post first dose of 9vHPV and 2vHPV vaccines, 99.4 to $100 \%$ and $100 \%$ of vaccinated girls and boys were seropositive to the vaccinated HPV types, respectively, whereas all individuals were seropositive to the HPV types included in the 9vHPV vaccine, regardless of the schedule used. These data show that flexible vaccination schedules can be safely used in the case of a vaccine shortage or change in national immunization program recommendations (21).

Current US guidelines recommend HPV vaccination for girls 11 to 12 years old with a catch-up vaccination for girls/women 13 to 26 years old; however, little is known about the population effectiveness of the existing catch-up vaccination recommendations. Hence, a nested case-control study on 4,357 CIN2+ cases and 21,773 matched controls was conducted, which confirmed that catch-up vaccination confers significant protection against incident $\mathrm{CIN}_{2}+$ and $\mathrm{CIN}_{3}+$ in women receiving at least three doses of the vaccine if they are 14 to 20 years old at the time of the first dose. Conversely, this was not true for women $\geq 21$ years old at the first dose. Whereas these findings support current catch-up vaccination guidelines for those that start the series up to age 20, additional research is needed to evaluate the effectiveness of such an approach in women 21 to 26 years old, especially in the light of recent calls to extend HPV vaccination to older women (22).

Results from the Costa Rica Vaccine Trial (23) and trials performed in India (24) implied that even a single dose of the 2vHPV or $4 \mathrm{vHPV}$ vaccine can induce sufficient long-lasting immunogenicity and effectiveness against incident HPV infection. Recent large-scale real-world data from Australia have confirmed this hypothesis: compared to unvaccinated women, the adjusted hazard ratios were significantly lower for vaccinated women, regardless of the number of doses received (0.65, 95\% CI 0.52-0.81; 0.61, 95\% CI $0.52-0.72$, and $0.59,95 \%$ CI $0.54-0.65$ for one, two, and three doses, respectively) (25). Similar results were also obtained in Denmark, where a one-dose HPV vaccination at age $\leq 16$ seemed to ensure similar protection as three doses (26). Because mounting evidence suggests the comparable effectiveness of a single dose to a two/three-dose HPV vaccination schedule in preventing high-grade cervical disease, one-dose HPV vaccination may be a viable strategy in the global elimination of cervical cancer, which would greatly reduce costs and requirements for national HPV vaccination programs (25). The hypothetical future reduction in the number of required HPV vaccine doses to a single dose was shown to be widely supported by immunization program stakeholders in low- and middle-income countries, provided that sufficient evidence on the immunological and clinical efficacy of such 
a strategy is obtained and a formal recommendation by the WHO is announced (27).

\section{HPV vaccination in immunocompromised individuals}

More data are becoming available on the long-term immunogenicity and safety of HPV vaccines in immunosuppressed populations, who bear the highest burden of HPV-related diseases. In a prospective observational cohort study of 4vHPV-vaccinated youth from the multicenter Pediatric HIV/AIDS Cohort Study Adolescent Master Protocol, HPV antibody titers were compared between perinatally HIV-infected (PHIV) and perinatally HIVexposed but uninfected (PHEU) youth. Interestingly, in PHIV, antibody titers were shown to be lower for all serotypes compared to PHEU. Higher GMTs were associated with lower HIV RNA viral load and higher CD4 cell count, suggesting that HPV vaccines may be less effective in PHIV children if given at a time of immunosuppression, asserting the need for HPV revaccination when immunocompetence is restored (28). Similar data were acquired from a cohort of perinatally HIV-1 infected sexually naive Kenyan boys and girls 9 to 14 years old. The study found that 7 months after receiving three doses of the 4VHPV vaccine, seroconversion to HPV6, HPV11, HPV16, and HPV18 occurred in 99.6\%, 97.2\%, $98.3 \%$, and $93.3 \%$ of the children, respectively. The vaccine was highly immunogenic with very few adverse events (AE) reported. Although HIV-1 infected children mounted a robust immune response that was comparable to other vaccinated populations regardless of their immune status, children with higher CD4 cell counts produced higher antibody titers than those with lower CD4 cell counts (29). The immunogenicity of the $4 \mathrm{VHPV}$ vaccine was also evaluated in a population of immunocompromised Australian children 5 to 18 years old with different underlying conditions. Following administration of three doses of the 4VHPV vaccine, $86.5 \%, 89.2 \%, 89.2 \%$, and $91.9 \%$ of children were seropositive at 60 months for HPV6, HPV11, HPV16 and HPV18, respectively, suggesting that a three-dose schedule using the $4 \mathrm{vHPV}$ vaccine remains immunogenic in this population for at least 5 years post vaccination (30).

It has been unclear whether HPV vaccines in HIV-infected adults are capable of establishing long-term immunity. Ellsworth et al. provide crucial data from a cohort of HIV-infected 4VHPVvaccinated adult males from the US, clearly showing that antibody titers peak 4 weeks after a third dose, followed by a gradual decrease in antibody titer levels. However, a delayed administration of the fourth dose of the $4 \mathrm{vHPV}$ vaccine induces a rapid increase in antibody titers, suggesting that standard three-dose series are capable of inducing immune memory and conferring long-term protection in HIV-infected men (31).

\section{Extension of indications for HPV vaccination: what is cost- effective?}

In light of the recent extension of vaccine indications, a literature review investigated the cost-effectiveness of vaccinating alternative populations. Whereas studies on men who have sex with men (MSM) consistently reported cost-effectiveness, HPV vaccination of heterosexual men would only be cost-effective if 9vHPV vaccine prices were reduced. In contrast, targeted vaccination of women over 26 is unlikely to be cost-effective (32). Authors have suggested that following the establishment of solid girls-only vaccination programs, targeted HPV vaccination of MSM should be the next priority in HPV prevention. However, these economic evaluations have important limitations when it comes to African countries. Given the disproportionate burden of HPV-related cancers in Africa and the time it could take to achieve herd immunity through girls-only HPV vaccination programs, there has also been a call for the introduction of gender-neutral HPV vaccination for national immunization programs in Africa (33).

\section{Real-life efficacy of HPV vaccines}

A decade has passed since the implementation of HPV vaccination, and post-licensing data from real-life settings are becoming available for increasing number of countries and populations. To date, more than 100 studies have evaluated the effectiveness and impact of HPV vaccines on preventing HPV infection and related disease (3). The largest systematic review and meta-analysis, which includes data from 60 million individuals and up to 8 years of follow-up after vaccination, summarizes the evidence and demonstrates the remarkable population-level impact of HPV vaccination on HPV infections, anogenital warts, and development of CIN2+ in women in real-world settings (34). The prevalence of HPV16 and HPV18 was found to decrease by $83 \%$ in girls 13 to 19 years old ( $\mathrm{RR} 0.17,95 \% \mathrm{CI} 0.11-0.25) 5$ to 6 years after vaccination, and by 66\% (RR 0.34, 95\% CI 0.23-0.49) in young women 20 to 24 years old. Substantial reductions in the incidence of anogenital warts were also observed: a $67 \%$ reduction (RR 0.33 , 95\% CI $0.24-0.46$ ) in girls, a 54\% reduction in young women (RR 0.46 , 95\% CI 0.36-0.60), a 48\% reduction in boys ( $\mathrm{RR} 0.2$, 95\% CI 0.370.75 ), and a $32 \%$ reduction ( $\mathrm{RR} 0.68,95 \% \mathrm{CI} 0.47-0.98$ ) in young men (34). Within less than a decade since HPV vaccination implementation, we are already noticing a $51 \%$ decrease in CIN2+ cases (RR 0.49, 95\% CI 0.42-0.58) in girls and a 31\% decrease in young women (RR 0.69, 95\% CI 0.57-0.84). The results of this meta-analysis provide substantial evidence of the impact of HPV vaccination and provide data on the real-world benefits of HPV vaccines in the prevention of cervical cancer with significant declines in high-risk (hr) HPV infection and CIN2+ (34). Although most studies on the real-world effect of HPV vaccination published in recent years have already been covered by the review by Drolet et al. (34), we additionally present some of the most intriguing data from individual countries.

\section{Decreasing incidence and prevalence of HPV types}

Australia was among the first countries to adopt primary HPVbased cervical screening, which for the first time made possible precise monitoring of HPV prevalence at a population level-an approach to HPV monitoring that used to be beyond the reach and budget of research studies (35). Data after the first 7 months of implementation showed 9.25\% (95\% CI: 9.09-9.42\%) prevalence of hrHPV among 116,052 primary screening samples, of which $2.14 \%$ (95\% CI: 2.05-2.22\%) were HPV16- or HPV18-positive. A peak in HPV prevalence was observed at 25 to 29 years of age, but this was due to non-vaccine HPV types. Across all ages, the prevalence of HPV16 or HPV18 remained low (35).

A decade since HPV vaccination introduction into their program, significant changes in cervical HPV prevalence have also been observed in Sweden. Although the HPV prevalence in women visiting a youth clinic in Stockholm, Sweden, in 2017-2018 was high (72.1\% for all HPV types and $65.1 \%$ for hrHPV), the prevalence of $27 \mathrm{hrHPV}$ was lower in vaccinated women (6o.1 vs. $86.73 \%, p$ 
= 0.006). Moreover, the prevalence of $4 \mathrm{VHPV}$ vaccine types was also considerably lower in the vaccinated women compared to unvaccinated women ( 5.8 vs. $26.7 \%, p=0.002$ ). Whereas $4 \mathrm{vHPV}$ vaccination has significantly decreased the vaccine type prevalence, several non-vaccine hrHPV types-namely, HPV39, HPV51, HPV56, and HPV59-remain relatively common (36). Similar data are reported from the United States, where the $4 \mathrm{vHPV}$ vaccine type prevalence decreased from $13.1 \%$ in 2007 to $2.9 \%$ in $2015-$ 2016 (prevalence ratio $=0.22 ; 95 \% \mathrm{CI} 0.17-0.29$ ), despite reaching only approximately 50\% coverage with two- to three-dose recommendations. A higher impact of HPV vaccination was observed among younger cohorts with a $78 \%$ decrease in prevalence among 20 - to 24 -year-old women and a $38 \%$ decrease in 25 - to 29 -yearold women. Declines were also observed in unvaccinated women, suggesting herd protection (37).

The observed declining trends of vaccine HPV types are not limited to cervical samples. In a cross-sectional study from the UK, various oral samples were tested for HPV DNA. The prevalence of oropharyngeal HPV16 in 940 study participants was significantly lower in vaccinated women compared to unvaccinated women (0.5\% vs 5.6\%, $p=0.04$ ). Interestingly, the HPV16 prevalence was shown to be lower in unvaccinated men than in unvaccinated women ( $0 \%$ vs 5.6\%, $p=0.08$ ), suggesting that herd immunity in males of the same age can be established even in female-only HPV vaccination programs (38).

The majority of data on HPV vaccine effectiveness in prevention of anal cancers are limited to males. In a study from the Netherlands that included 548 women 16 to 24 years old, the vaccine effectiveness against anal HPV16 or HPV18 positivity was $89.9 \%$ (95\% CI, 63.0\%-97.2), demonstrating high effectiveness of the vaccine for up to 8 years, which is comparable to effectiveness against genital infections (39).

\section{Decreasing incidence and prevalence of anogenital warts}

Because anogenital warts usually develop 2 to 3 months after infection, the impact of HPV vaccines on the incidence of anogenital warts provides early evidence of vaccination efficacy. In a recent systematic review from the United States, the authors assessed the real-life impact of HPV vaccines on anogenital warts. Consistent declines in diagnoses of anogenital warts were observed in females 25 and younger following vaccination implementation in 2006 in the United States. In males, the same trend was observed-albeit to a lesser degree, most likely due to herd immunity-until 2011, when male vaccination began, with declines becoming even more evident. In contrast, no trends were observed among people over 25 (40). These data differ somewhat from the results obtained by Mann et al. (41), who analyzed data from sexually transmitted disease clinics in the United States. Declining temporal trends in the prevalence of anogenital warts from 2010 to 2016 were observed not only in women under 40, but also in men who have sex with women only and are younger than 40, and MSM in all age categories (41). Interestingly, a similar declining trend was also observed in older age groups, which is unlikely to be a result of HPV vaccination. Similar data on declining trends in the prevalence of anogenital warts among patients attending sexual health clinics have been reported from England, where the 2 vHPV vaccine was introduced in 2008 , followed by the introduction of the $4 \mathrm{VHPV}$ vaccine in 2012. An $82.3 \%$ decline was observed among females 15 to 17 years old and a $67.7 \%$ decline in men of the same age between 2014 and 2017 (42).

\section{Decreasing rates of precancerous lesions}

Currently, the most proximal outcome to cervical cancer is precancerous lesions (CIN2+), which typically develop a few years after primary HPV infection. More real-life data are becoming available on the impact of HPV vaccination on the development of CIN2+, confirming early findings of randomized controlled trials showing the efficacy of HPV vaccines for preventing cervical dysplasia $(43,44)$.

One of the most dramatic reductions in preneoplastic cervical lesions was observed in Scotland, which has had consistently high (above $85 \%$ ) 2vHPV vaccine coverage rates among routinely immunized cohorts during the last 10 years. This led to an $89 \%$ reduction (95\% CI, 81-94\%; from $0.59 \%$ [95\% CI, $0.48-0.71 \%$ ] to $0.06 \%$ [95\% CI, 0.04-0.11\%]) in prevalent $\mathrm{CIN}_{3}+$ and an $88 \%$ reduction (95\% CI, 83-92\%; from 0.69\% [95\% CI, 0.58-0.63\%] to $0.15 \%$ [95\% CI, 0.10-0.21\%]) in CIN2+ among vaccinated women born in 1995 and 1996, compared to unvaccinated women born in 1988. Moreover, there was evidence of clinically relevant herd protection in unvaccinated women (45).

In New Zealand, the 4vHPV vaccine has been available since 2008. The proportion of newly diagnosed CIN2 attributable to HPV16/18 infection was observed over time, using partial genotyping of samples from women 17 to 24 years old, with biopsydiagnosed CIN2 enrolled in a prospective CIN2 observational management trial (PRINCess). From 2013 to 2016, the proportion of women whose liquid-based cytology samples were HPV16- or HPV18-positive decreased from $43 \%$ to $13 \%$. Although vaccinated women had low rates of HPV16- and HPV18-positive CIN2 lesions in the time period observed, a substantial decrease from $66 \%$ to $17 \%$ in $\mathrm{HPV} 16 / 18$ positivity was documented in unvaccinated women, likely due to a herd effect (46).

In the US, the $4 \mathrm{vHPV}$ vaccine was most commonly administered from 2006 to 2015 , followed by the 9vHPV vaccine, which is the only available HPV vaccine in the country since 2017. Using archived specimens from women 18 to 39 years old diagnosed with $\mathrm{CIN} 2+$ and data from a population-based surveillance system (the HPV Vaccine Impact Monitoring Project), the proportion of CIN2+ by HPV type was assessed over time. The proportion of HPV16- or HPV18-positive CIN2+ declined from 52.7\% in 2008 to $44.1 \%$ in 2014 (47). Substantial declines in the proportion of HPV16- and HPV18-positive CIN2+ were recorded among women that were vaccinated (55.2-33.3\%). Furthermore, a decline was also observed in unvaccinated women (51.0-47.3\%), again suggesting the establishment of herd protection against CIN2+ (47).

\section{No evidence of HPV type replacement following HPV vaccination}

Despite initial fears that HPV type replacement would occur following HPV vaccination implementation, similar to what was observed with the pneumococcal vaccine, there is a lack of evidence of such a phenomenon with respect to HPV (1). Moreover, an 11year follow-up on US women showed a $45.8 \%$ decrease in the prevalence of HPV types genetically related to HPV16, demonstrating evidence of cross-protection following $4 \mathrm{VHPV}$ vaccination, whereas no statistically significant changes in the prevalence of HPV types that are genetically unrelated to $4 \mathrm{vHPV}$ vaccine types was observed, suggesting that HPV type replacement has not occurred (48). However, as shown by Gray et al. (49), some HPV types may become more common in groups exhibiting sexual risk-taking be- 
havior, suggesting that continued post-vaccination surveillance (especially for HPV51 and HPV52) may be warranted.

\section{HPV vaccine safety data: an update}

Despite being labeled extremely safe by the WHO, HPV vaccines have been falsely and unfairly associated with numerous AEs and dubious syndromes. Heightened reporting by the media has significantly impacted already established immunization programs in various countries, including but not limited to Denmark, Japan, and Colombia. A retrospective observational study analyzing clusters of AE reports in Denmark identified four clusters, with fatigue, dizziness, and headache as the most common symptoms reported. These symptoms were mainly non-specific and tended to occur commonly in the targeted population. Moreover, there was no evidence that these clusters represented chronic fatigue syndrome (CFS) or any novel autonomic dysfunction syndrome. Nevertheless, an anomalous spike in submissions of AE reports (mostly cluster 2) seemed to result from stimulated reporting on symptoms of CFS and postural orthostatic tachycardia syndrome (POTS), further highlighting the influence of negative media reporting on HPV vaccination coverage (50). Worryingly, an overview of reports of long-lasting fatigue following 2 vHPV vaccination published in 2013 and updated in 2015 that was picked up by the Dutch media inevitably led to further reports on this syndrome. Hence, a retrospective cohort study on 69,429 12- to 16-year-old Dutch girls was conducted to investigate these allegations. The study found that long-lasting fatigue is common among adolescent girls, whereas CFS is rarely diagnosed with no statistically significant increase in incidence rates during the post-vaccination period compared to the pre-vaccination period (51).

In response to lingering public concerns that HPV vaccine causes autoimmune diseases, a retrospective cohort study evaluating the association between HPV vaccination and diabetes mellitus type 1 on 911,648 individuals was conducted. No increased risk of diabetes mellitus type 1 correlated with HPV vaccination (hazard ratio 1.21, 95\% CI 0.94-1.57) was found over the 10 years of the study period (52). Furthermore, a recent systemic review and meta-analysis on more than 169,000 autoimmune disorder events further confirmed that HPV vaccination is not associated with an increased risk for subsequent autoimmune diseases (odds ratio [OR] 1.00, 95\% CI 0.95-1.06), particularly among individuals with preexisting autoimmune conditions (OR 0.82, 95\% CI 0.70-0.96) (53).

Similar to previously published data, an analysis of reports to the US Vaccine Adverse Event Reporting System (VAERS) of AE following 2vHPV vaccination between 2009 and 2017 found no new or unexpected safety concerns with the use of the $2 \mathrm{vHPV}$ vaccine (54).

Because those opposing HPV vaccination in Japan claim that there are no vaccine efficacy or safety data to clearly show that HPV vaccines work in a Japanese population, results from several trials on Japanese men and women were recently published to bridge the findings from international trials on immunogenicity, safety, and efficacy (55-57). Thus, the 4vHPV vaccine was found to be immunogenic, safe, and well tolerated in Japanese boys (56) and men (57), whereas the 2vHPV vaccine was shown to be highly effective against targeted HPV types and conferred crossprotection against HPV31, HPV45, and HPV52 up to 6 years post vaccination (55). Hopefully, these data will finally convince Japanese politicians to reinstate proactive recommendations for HPV vaccination, which could to some degree revert the catastrophic decline in HPV vaccination rates following suspension of these recommendations in 2013 (55).

\section{No change in sexual behavior following HPV vaccination}

Although some groups expressed concern that HPV vaccination will result in an increase in sexual risk-taking behaviors among adolescents, there is no evidence that HPV vaccination leads to increased promiscuity or unsafe sexual practices. This has been additionally confirmed on a large study evaluating population-level changes in sexual behaviors prior to and after implementation of school-based HPV vaccination program in British Columbia, Canada. Data on 298,265 heterosexual girls showed that sexual risk behaviors reported by adolescent girls following implementation of HPV vaccination either stayed the same (e.g., number of sexual partners reported) or declined. Thus, significant declines in the proportion of girls reporting ever having sexual intercourse (OR 0.79), having sexual intercourse before age 14 (OR 0.76), and reported substance use before intercourse (OR 0.69) were found in the period from 2003 to 2013 (58). Similar trends were also shown for college-age men and women from the US (59) and Danish pupils (6o). Nevertheless, HPV-vaccinated adolescents from Denmark were less likely to use a condom despite their higher sexually transmitted infections (STI) awareness; however, this trend is probably unrelated to HPV vaccination itself (6o).

\section{Novel prophylactic HPV vaccines and innovative technologies for HPV vaccine production}

Despite being highly effective in preventing HPV infections, the 2vHPV and 4vHPV vaccines confer protection against a limited array of HPV types and are associated with high production and delivery costs. To address these issues, research is focusing on finding alternative and/or innovative methods to produce and deliver novel HPV vaccines. Whereas the 9vHPV vaccine may be considered the first of a new HPV vaccine generation, several other second-generation HPV vaccines are currently undergoing preclinical and clinical evaluations.

Instead of using eukaryotic systems, which are expensive to manufacture, HPV L1 virus-like particles (VLPs) can be produced at much lower costs using bacteria. Two Escherichia coli-based prophylactic HPV vaccines against HPV16/18 and HPV6/11 are currently in phase III and phase I clinical trials, respectively (61). To decrease the production costs, expression systems of the methylotrophic yeasts Pichia pastoris and Hansenula polymorpha are also being used to produce HPV6, HPV11, HPV16, and HPV18 VLPs. These types of vaccines are undergoing phase I trials (61). Other advanced systems not presented in detail due to the scope of this review include transgenic plants and attenuated bacteria (e.g., mutant Salmonella enterica or Shigella) that are capable of producing L1 VLPs, and recombinant viral vaccines (e.g., recombinant baculovirus) containing the HPV16 L1 gene (62).

Due to the fact that the L2 protein induces broad cross-neutralizing antibodies, it was thought to be the ideal candidate for generating a so-called pan-HPV vaccine. However, the suboptimal immunogenicity and limited neutralizing spectrum of L2 seemed to be the major caveat in developing efficient prophylactic L2-based HPV vaccines. This might potentially change because a recent study showed that rationally designed flagellin-L2 fusion peptides (Fla-5PcL2) were capable of inducing robust broad- 
spectrum serum (when injected intramuscularly) and mucosal (when injected intranasally) neutralizing antibodies. Moreover, both routes of vaccine administration induced potent protection against vaginal pseudovirus challenges in mice, suggesting that Fla-5PcL2 is a promising L2-based HPV vaccine candidate (63).

The pentameric subunits or capsomeres of the L1 protein are especially attractive for development of affordable second-generation HPV vaccines. In contrast to VLPs that are composed of 360 copies of the L1, the capsomeres only contain five L1 monomers, expressing all essential neutralizing epitopes capable of inducing immune responses. They are easily produced in $E$. coli and, because capsomeres can be lyophilized and consequently shipped as well as stored without refrigeration $(61,62)$, this could significantly improve the availability of HPV vaccine in developing countries. Chinese researchers recently evaluated a novel HPV16 L1 pentamer-loaded hybrid particles vaccine system, showing the importance of particle size when developing novel HPV vaccines, with HPV L1 capsomer particle size of $1 \mu \mathrm{m}$ generating the strongest immune reactions in BALB/c mice (64).

In addition to its cost, the requirement for continuous refrigeration (between 2 and $8{ }^{\circ} \mathrm{C}$ ) sets another barrier that hinders widespread availability of the HPV vaccine, especially in remote regions of the world. Data on immunogenicity and efficacy of spray-dried 9vHPV vaccines are encouraging, showing considerable protection of such thermostable vaccine against HPV infection in mice following storage at temperatures up to $40{ }^{\circ} \mathrm{C}$ for 3 months. Moreover, it even seems that the 9vHPV vaccine, as currently marketed, could be stored and transported at temperatures above $8{ }^{\circ} \mathrm{C}$ without losing efficacy, which would be especially convenient in low and middle-income countries (65).

Whereas vaccines are mostly injected intramuscularly, intradermal administration can be an alternative route of vaccine delivery with several advantages: for example, the antigens can be applied at a lower dosage and may be capable of inducing an immune response in intramuscularly vaccinated non-responders. Results from the first evaluation of a high-density microprojection vaccine patch (Nanopatch ${ }^{\mathrm{TM}}$ ) coated with unadjuvanted HPV vaccine showed that this type of HPV vaccine remained stable during cold shipping, was delivered effectively, and was capable of generating an immune response in rhesus macaques (66).

\section{The future of HPV vaccination}

The impact of HPV vaccines has by far exceeded our initial expectations; however, their potential is still largely unexploited. Unfortunately, despite having access to HPV vaccination, many women are still being diagnosed with high-grade cervical lesions, among them also vaccine-eligible women that were not vaccinated. Surprisingly, the three most common self-reported reasons for not receiving the vaccine among vaccine-eligible women diagnosed with $\mathrm{CIN} 2+$ from the US were age (e.g., being too old to receive the vaccine; $31.3 \%$ ), lack of provider recommendation for vaccination (19.9\%), and previous diagnosis of HPV infection (17.5\%). These results suggest the need to dispel myths about HPV vaccine eligibility-older age and previous diagnosis of HPV infection are not contraindications for HPV vaccination-and to highlight the importance of providers in promotion of vaccination for all eligible patients (67).

An example of an innovative intervention toward increased HPV vaccination coverage is the FASTER strategy, which offers HPV vaccination to all women between 25 and 45 years old at- tending cervical cancer screening (68). Encouraging data are already available for Mexico, where almost all eligible women $(3,282 / 3,474 ; 93 \%)$ accepted HPV vaccination as part of a combined screening and HPV vaccination program (69).

The idea of combinatorial vaccines that would simultaneously protect against HPV and other diseases also became an attractive approach toward improving vaccination coverage rates. Recently, a proof-of-concept combinatorial vaccine using a VLP displaying an HPV RG1 epitope and VAR2CSA placental malaria antigen proved to be able to reduce HPV infection while inducing anti-VAR2CSA IgG antibodies in mice. Such a vaccine with dual specificity would be especially convenient in Sub-Saharan Africa, where the target population for vaccination against both HPV and placental malaria (e.g., adolescent girls) basically overlaps (70). To achieve elimination of rubella while potentially increasing HPV vaccine uptake in developing countries, a combinatorial HPV16/18 and rubella vaccine was designed. Interestingly, immunized mice developed specific antibodies against both HPV and rubella that were even higher than in mice immunized with only rubella or HPV vaccine (71). Moreover, results from a randomized controlled trial showed feasibility of concomitant administration of the 4vHPV vaccine and tetanus, diphtheria, pertussis (Tdap) vaccine with quadrivalent meningococcal CRM197-conjugate vaccine (MenACWY-CRM) in adolescents. Such concomitant administration of different vaccines could maximize the opportunity for improving community protection and compliance with scheduled vaccination (72).

A large study of 18,247 formalin-fixed paraffin-embedded specimens from 50 countries highlighted the potential impact of the 9vHPV vaccine in preventing HPV-related disease, suggesting that the addition of the five high-risk HPV types (HPV31, HPV 33 , HPV 45, HPV52, HPV 58) to HPV6, HPV11, HPV16, and HPV18 in the 9vHPV vaccine could potentially prevent approximately 90\% of cervical cancer cases and globally reduce 50\% of all HPV-related cancer cases (73). Moreover, a meta-analysis by Drolet et al. (34) that evaluated data from 65 studies on more than 60 million HPV-vaccinated individuals with up to an 8-year follow-up provided the most up-to-date information on HPV vaccine impact at a population level. Most importantly, Drolet et al. have shown that, compared to countries with single-cohort HPV vaccination and/ or low coverage rates, countries that adopted multiple age cohorts for HPV vaccination schedules tend to observe faster and higherimpact results, including benefits due to herd effect (34).

The success of HPV vaccines has drastically reframed our initial goals, moving from cervical cancer prevention to cervical cancer elimination. However, multi-level interventions based on a combination of HPV vaccination, screening, and treatment of cervical disease will have to be tightly coordinated and supported by national and/or regional healthcare officials and authorities in order to approach the cervical cancer elimination threshold. Australia, a pioneer in introducing a successful national HPV vaccination program with high and consistent coverage rates across both sexes, will mostly likely be one of the first countries to reach elimination thresholds; the estimates predict that the age-standardized annual incidence of cervical cancer will drop below six new cases per 100,000 women by 2020, and fewer than four new cases per 100,000 women by 2028 . With a combination of regular cervical cancer screening, treatment of preneoplastic lesions, and high HPV vaccination coverage rates, cervical cancer will probably be eliminated as a public health problem in Australia within the next 20 years (74). A modeling study performed by Simms et 
al. (75) further elucidates the requirements for elimination of cervical cancer. In the absence of further interventions, an estimated 44.4 million cervical cancer cases would be diagnosed at the global level over the period from 2020 to 2069 , with almost two-thirds of cases occurring in low- or middle-income countries. However, if widespread high-coverage HPV vaccination in combination with regular cervical cancer screening is adopted from 2020 onward, we could also achieve elimination thresholds of fewer than four cases of cervical cancer per 100,000 women per year in low-income countries by the end of the century, and this effect would be achieved a few decades earlier in high-income countries (by 2065-2069) and in middle-income countries (by 2070-2079) (75).

\section{Conclusions}

Additional evidence contributes to the already overwhelming data on HPV vaccine safety, durability of immune protection, and real-life effectiveness. Unfortunately, HPV vaccines will inevitably experience obstacles despite their substantial success, with the most threatening issue currently being the HPV vaccine shortage. Potential barriers that prevent worldwide implementation of gender-neutral HPV vaccination should be addressed and acted upon immediately in order to exploit the incredible potential of prophylactic HPV vaccines in time.

\section{References}

1. Šterbenc A, Maver PJ, Poljak M. Recent advances in prophylactic human papillomavirus (HPV) vaccination: a review of key literature published between September 2017 and September 2018. Acta Dermatovenerol Alp Pannonica Adriat. 2018;27:193-201.

2. Maver PJ, Poljak M. Progress in prophylactic human papillomavirus (HPV) vaccination in 2016: a literature review. Vaccine. 2018;36:5416-23.

3. de Sanjose S, Brotons M, LaMontagne DS, Bruni L. Human papillomavirus vaccine disease impact beyond expectations. Curr Opin Virol. 2019;39:16-22.

4. U.S. Food \& Drug Administration. FDA approves expanded use of Gardasil 9 to include individuals 27 through 45 years old [Internet]. [cited 2019 June 2]. Available from: https://www.fda.gov/news-events/press-announcements/fdaapproves-expanded-use-gardasil-9-include-individuals-27-through-45-yearsold.

5. Meites E, Szilagyi PG, Chesson HW, Unger ER, Romero JR, Markowitz LE. Human papillomavirus vaccination for adults: updated recommendations of the Advisory Committee on Immunization Practices. MMWR Morb Mortal Wkly Rep. 2019; 68:698-702.

6. Joura EA, Kyrgiou M, Bosch FX, Kesic V, Niemenen P, Redman CW, et al. Human papillomavirus vaccination: the ESGO-EFC position paper of the European Society of Gynaecologic Oncology and the European Federation for Colposcopy. Eur J Cancer. 2019;116:21-6.

7. European Centre for Disease Prevention and Control. Public consultation on draft guidance for introduction of HPV vaccines in EU countries: focus on 9-valent HPV vaccine and vaccination of boys and people living with HIV [Internet]. Stockholm; 2019. [cited 2019 May 2]. Available from: https://ecdc.europa.eu/ sites/portal/files/documents/hpv-public-consultation-3-April.pdf.

8. Wright TC Jr, Parvu V, Stoler MH, Kodsi S, Eckert K, Yanson K, et al. HPV infections and cytologic abnormalities in vaccinated women 21-34 years of age: results from the baseline phase of the Onclarity trial. Gynecol Oncol. 2019;153:259-65.

9. Giuliano AR, Joura EA, Garland SM, Huh WK, Iversen OE, Kjaer SK, et al. Ninevalent HPV vaccine efficacy against related diseases and definitive therapy: comparison with historic placebo population. Gynecol Oncol. 2019;154:110-7.

10. Ghelardi A, Parazzini F, Martella F, Pieralli A, Bay P, Tonetti A, et al. SPERANZA project: HPV vaccination after treatment for CIN2. Gynecol Oncol. 2018;151:229-34.

11. Stankiewicz Karita HC, Hauge K, Magaret A, Mao C, Schouten J, Grieco V, et al. Effect of human papillomavirus vaccine to interrupt recurrence of vulvar and anal neoplasia (VIVA): a trial protocol. JAMA Netw Open. 2019;2:e190819.

12. Artemchuk H, Eriksson T, Poljak M, Surcel HM, Dillner J, Lehtinen M, et al. Longterm antibody response to human papillomavirus vaccines: up to 12 years of follow-up in the Finnish maternity cohort. J Infect Dis. 2019;219:582-9.

13. Lazcano-Ponce E, Torres-Ibarra L, Cruz-Valdez A, Salmerón J, Barrientos-Gutiérrez T, Prado-Galbarro J, et al. Persistence of immunity when using different human papillomavirus vaccination schedules and booster-dose effects 5 years after primary vaccination. J Infect Dis. 2019;219:41-9.

14. Folschweiller N, Behre U, Dionne M, Durando P, Esposito S, Ferguson L, et al. Long-term cross-reactivity against nonvaccine human papillomavirus types 31 and 45 after 2- or 3-dose schedules of the ASo4-adjuvanted human HPV-16/18 vaccine. J Infect Dis. 2019;219:1799-803.

15. Parker KH, Kemp TJ, Isaacs-Soriano K, Abrahamsen M, Pan Y, Lazcano-Ponce E, et al. HPV-specific antibodies at the oral cavity up to 30 months after the start of vaccination with the quadrivalent HPV vaccine among mid-adult aged men. Vaccine. 2019;37:2864-9.

16. World Health Organization. Human papillomavirus vaccine: WHO position paper, May 2017. Weekly Epidemiol Rec. 2017;92:241-98.

17. Blomberg M, Dehlendorff C, Sand C, Kjaer SK. Dose-related differences in effectiveness of human papillomavirus vaccination against genital warts: a nationwide study of 550,000 young girls. Clin Infect Dis. 2015;61:676-82.
18. Herweijer E, Leval A, Ploner A, Eloranta S, Simard JF, Dillner J, et al. Association of varying number of doses of quadrivalent human papillomavirus vaccine with incidence of condyloma. JAMA. 2014;311:597-603.

19. Dehlendorff C, Sparén P, Baldur-Felskov B, Herweijer E, Arnheim-Dahlström L, Ploner A, et al. Effectiveness of varying number of doses and timing between doses of quadrivalent HPV vaccine against severe cervical lesions. Vaccine. 2018;36:6373-8.

20. Basu P, Muwonge R, Bhatla N, Nene BM, Joshi S, Esmy PO, et al. Two-dose recommendation for human papillomavirus vaccine can be extended up to 18 years-updated evidence from Indian follow-up cohort study. Papillomavirus Res. 2019;7:75-81.

21. Gilca V, Sauvageau C, Panicker G, De Serres G, Ouakki M, Unger ER. Immunogenicity and safety of a mixed vaccination schedule with one dose of nonavalent and one dose of bivalent HPV vaccine versus two doses of nonavalent vaccine-a randomized clinical trial. Vaccine. 2018;36:7017-24.

22. Silverberg MJ, Leyden WA, Lam JO, Gregorich SE, Huchko MJ, Kulasingam S, et al. Effectiveness of catch-up human papillomavirus vaccination on incident cervical neoplasia in a US health-care setting: a population-based case-control study. Lancet Child Adolesc Health. 2018;2:707-14.

23. Safaeian M, Sampson JN, Pan Y, Porras C, Kemp TJ, Herrero R, et al. Durability of protection afforded by fewer doses of the HPV16/18 vaccine: the CVT trial. J Natl Cancer Inst. 2018;110:205-12.

24. Sankaranarayanan R, Joshi S, Muwonge R, Esmy PO, Basu P, Prabhu P, et al. Can a single dose of human papillomavirus (HPV) vaccine prevent cervical cancer? Early findings from an Indian study. Vaccine. 2018;36:4783-91.

25. Brotherton JM, Budd A, Rompotis C, Bartlett N, Malloy MJ, Andersen RL, et al. Is one dose of human papillomavirus vaccine as effective as three? A national cohort analysis. Papillomavirus Res. 2019;8:100177.

26. Verdoodt F, Dehlendorff C, Kjaer SK. Dose-related effectiveness of quadrivalent human papillomavirus vaccine against cervical intraepithelial neoplasia: a Danish nationwide cohort study. Clin Infect Dis. 2019. [Epub ahead of print].

27. Gallagher KE, Kelly H, Cocks N, Dixon S, Mounier-Jack S, Howard N, et al. Vaccine programme stakeholder perspectives on a hypothetical single-dose human papillomavirus (HPV) vaccine schedule in low and middle-income countries. Papillomavirus Res. 2018;6:33-40.

28. Moscicki AB, Karalius B, Tassiopoulos K, Yao TJ, Jacobson DL, Patel K, et al. Human papillomavirus antibody levels and quadrivalent vaccine clinical effectiveness in perinatally human immunodeficiency virus-infected and exposed, uninfected youth. Clin Infect Dis. 2019;69:1183-91.

29. Mugo NR, Eckert L, Magaret AS, Cheng A, Mwaniki L, Ngure K, et al. Quadrivalent HPV vaccine in HIV-1-infected early adolescent girls and boys in Kenya: month 7 and 12 post vaccine immunogenicity and correlation with immune status. Vaccine. 2018;36:7025-32.

30. MacIntyre CR, Shaw PJ, Mackie FE, Boros C, Marshall H, Seale H, et al. Long term follow up of persistence of immunity following quadrivalent human papillomavirus (HPV) vaccine in immunocompromised children. Vaccine. 2019;37:5630-6.

31. Ellsworth GB, Lensing SY, Ogilvie CB, Lee JY, Goldstone SE, Berry-Lawhorn JM, et al. A delayed dose of quadrivalent human papillomavirus vaccine demonstrates immune memory in HIV-1-infected men. Papillomavirus Res. 2018;6:11-4.

32. Soe NN, Ong JJ, Ma X, Fairley CK, Latt PM, Jing J, et al. Should human papillomavirus vaccination target women over age 26 , heterosexual men and men who have sex with men? A targeted literature review of cost-effectiveness. Hum Vaccin Immunother. 2018;14:3010-8.

33. Chido-Amajuoyi OG, Domgue JF, Obi-Jeff C, Schmeler K, Shete S. A call for the introduction of gender-neutral HPV vaccination to national immunisation programmes in Africa. Lancet Glob Health. 2019;7:e20-e21. 
34. Drolet M, Bénard É, Pérez N, Brisson M, Ali H, Boily M-C, et al. Population-level impact and herd effects following the introduction of human papillomavirus vaccination programmes: updated systematic review and meta-analysis. The Lancet. 2019;394:497-509.

35. Brotherton JML, Hawkes D, Sultana F, Malloy MJ, Machalek DA, Smith MA, et al. Age-specific HPV prevalence among 116,052 women in Australia's renewed cervical screening program: a new tool for monitoring vaccine impact. Vaccine. 2019;37:412-6.

36. Ährlund-Richter A, Cheng L, Hu YOO, Svensson M, Pennhag AAL, Ursu RG, et al. Changes in cervical human papillomavirus (HPV) prevalence at a youth clinic in Stockholm, Sweden, a decade after the introduction of the HPV vaccine. Front Cell Infect Microbiol. 2019;9:59.

37. Markowitz LE, Naleway AL, Lewis RM, Crane B, Querec TD, Weinmann S, et al. Declines in HPV vaccine type prevalence in women screened for cervical cancer in the United States: evidence of direct and herd effects of vaccination. Vaccine. 2019;37:3918-24.

38. Mehanna H, Bryant TS, Babrah J, Louie K, Bryant JL, Spruce RJ, et al. Human papillomavirus (HPV) vaccine effectiveness and potential herd immunity for reducing oncogenic oropharyngeal HPV-16 prevalence in the United Kingdom: a cross-sectional study. Clin Infect Dis. 2019;69:1296-302.

39. Woestenberg PJ, King AJ, Van Benthem BHB, Leussink S, Van der Sande MAB, Hoebe CJPA, et al. Bivalent vaccine effectiveness against anal human papillomavirus positivity among female sexually transmitted infection clinic visitors in the Netherlands. J Infect Dis. 2018;217:213-22.

40. Yakely AE, Avni-Singer L, Oliveira CR, Niccolai LM. Human papillomavirus vaccination and anogenital warts: a systematic review of impact and effectiveness in the United States. Sex Transm Dis. 2019;46:213-20.

41. Mann LM, Llata E, Flagg EW, Hong J, Asbel L, Carlos-Henderson J, et al. Trends in the prevalence of anogenital warts among patients at sexually transmitted disease clinics-Sexually Transmitted Disease Surveillance Network, United States, 2010-2016. J Infect Dis. 2019;219:1389-97.

42. Checchi M, Mesher D, Mohammed H, Soldan K. Declines in anogenital warts diagnoses since the change in 2012 to use the quadrivalent HPV vaccine in England: data to end 2017. Sex Transm Infect. 2019;95:368-73.

43. FUTURE II Study Group. Quadrivalent vaccine against human papillomavirus to prevent high-grade cervical lesions. N Engl J Med. 2007;356:1915-27.

44. Paavonen J, Naud P, Salmerón J, Wheeler C, Chow S-N, Apter D, et al. Efficacy of human papillomavirus (HPV)-16/18 ASo4-adjuvanted vaccine against cervical infection and precancer caused by oncogenic HPV types (PATRICIA): final analysis of a double-blind, randomised study in young women. The Lancet. 2009; 374:301-14.

45. Palmer T, Wallace L, Pollock KG, Cuschieri K, Robertson C, Kavanagh K, et al. Prevalence of cervical disease at age 20 after immunisation with bivalent HPV vaccine at age $12-13$ in Scotland: retrospective population study. BMJ. 2019; $365: \operatorname{l1} 161$.

46. Innes CR, Sykes PH, Harker D, Williman JA, Van der Griend RA, Whitehead M, et al. Changes in human papillomavirus genotypes associated with cervical intraepithelial neoplasia grade 2 lesions in a cohort of young women (20132016). Papillomavirus Res. 2018;6:77-82.

47. McClung NM, Gargano JW, Bennett NM, Niccolai LM, Abdullah N, Griffin MR, et al. Trends in human papillomavirus vaccine types 16 and 18 in cervical precancers, 2008-2014. Cancer Epidemiol Prev Biomark. 2019;28:602-9.

48. Covert C, Ding L, Brown D, Franco EL, Bernstein DI, Kahn JA. Evidence for crossprotection but not type-replacement over the 11 years after human papillomavirus vaccine introduction. Hum Vaccin Immunother. 2019;15:1962-9.

49. Gray P, Luostarinen T, Vänskä S, Eriksson T, Lagheden C, Man I, et al. Occurrence of human papillomavirus (HPV) type replacement by sexual risk-taking behaviour group: post-hoc analysis of a community randomized clinical trial up to 9 years after vaccination (IV). Int J Cancer. 2019;145:785-96.

50. Ward D, Thorsen NM, Frisch M, Valentiner-Branth P, Mølbak K, Hviid A. A cluster analysis of serious adverse event reports after human papillomavirus (HPV) vaccination in Danish girls and young women, September 2009 to August 2017. Euro Surveill. 2019;24.

51. Schurink-Van't Klooster TM, Kemmeren JM, van der Maas NAT, van de Putte EM, Ter Wolbeek M, Nijhof SL, et al. No evidence found for an increased risk of longterm fatigue following human papillomavirus vaccination of adolescent girls. Vaccine. 2018;36:6796-802.

52. Klein NP, Goddard K, Lewis E, Ross P, Gee J, DeStefano F, et al. Long term risk of developing type 1 diabetes after HPV vaccination in males and females. Vaccine. 2019;37:1938-44.

53. Jiang HY, Shi YD, Zhang X, Pan LY, Xie YR, Jiang CM, et al. Human papillomavirus vaccination and the risk of autoimmune disorders: a systematic review and meta-analysis. Vaccine. 2019;37:3031-9.
54. Suragh TA, Lewis P, Arana J, Mba-Jonas A, Li R, Stewart B, et al. Safety of bivalent human papillomavirus vaccine in the US vaccine adverse event reporting system (VAERS), 2009-2017. Br J Clin Pharmacol. 2018;84:2928-32.

55. Kudo R, Yamaguchi M, Sekine M, Adachi S, Ueda Y, Miyagi E, et al. Bivalent human papillomavirus vaccine effectiveness in a Japanese population: high vac cine-type-specific effectiveness and evidence of cross-protection. J Infect Dis. 2019;219:382-90.

56. Murata S, Takeuchi Y, Yamanaka K, Hayakawa J, Yoshida M, Yokokawa R, et al. Safety and immunogenicity of the quadrivalent HPV vaccine in Japanese boys: a phase 3, open-label study. Jpn J Infect Dis. 2019. [Epub ahead of print].

57. Mikamo H, Yamagishi Y, Murata S, Yokokawa R, Han SR, Wakana A, et al. Efficacy, safety, and immunogenicity of a quadrivalent HPV vaccine in Japanese men: a randomized, phase 3, placebo-controlled study. Vaccine. 2019;37:1651-8.

58. Ogilvie GS, Phan F, Pedersen HN, Dobson SR, Naus M, Saewyc EM. Populationlevel sexual behaviours in adolescent girls before and after introduction of the human papillomavirus vaccine (2003-2013). CMAJ. 2018;190:E1221-E1226.

59. Brouwer AF, Delinger RL, Eisenberg MC, Campredon LP, Walline HM, Carey TE, et al. HPV vaccination has not increased sexual activity or accelerated sexual debut in a college-aged cohort of men and women. BMC Public Health. 2019;19:821.

6o. Svarrer RO, Rasmussen AL, Lauszus FF, Hammer A. No effect of human papillomavirus vaccination on sexual debut of school children. Dan Med J. 2019;66.

61. Barra F, Leone Roberti Maggiore U, Bogani G, Ditto A, Signorelli M, Martinelli F, et al. New prophylactics human papilloma virus (HPV) vaccines against cervical cancer. J Obstet Gynaecol. 2019;39:1-10.

62. Dadar M, Chakraborty S, Dhama K, Prasad M, Khandia R, Hassan S, et al. Advances in designing and developing vaccines, drugs and therapeutic approaches to counter human papilloma virus. Front Immunol. 2018;9:2478.

63. Zhang T, Chen X, Liu H, Bao Q, Wang Z, Liao G, et al. A rationally designed flagellin-L2 fusion protein induced serum and mucosal neutralizing antibodies against multiple HPV types. Vaccine. 2019;37:4022-30.

64. Jia C, Yang T, Liu Y, Zhu A, Yin F, Wang Y, et al. A novel human papillomavirus $16 \mathrm{~L}$ pentamer-loaded hybrid particles vaccine system: influence of size on immune responses. ACS Appl Mater Interfaces. 2018;10:35745-59.

65. Kunda NK, Peabody J, Zhai L, Price DN, Chackerian B, Tumban E, et al. Evaluation of the thermal stability and the protective efficacy of spray-dried HPV vaccine, Gardasil ${ }^{\circledR}$ 9. Hum Vaccin Immunother. 2019;15:1995-2002.

66. Meyer BK, Kendall MAF, Williams DM, Bett AJ, Dubey S, Gentzel RC, et al. Immune response and reactogenicity of an unadjuvanted intradermally delivered human papillomavirus vaccine using a first generation Nanopatch ${ }^{\mathrm{TM}}$ in rhesus macaques: an exploratory, pre-clinical feasibility assessment. Vaccine: $\mathrm{X}$. 2019;2:100030.

67. Russ SM, Brackney M, Meek J, Niccolai LM. Missed opportunities for HPV vaccination among vaccine-eligible women with high grade cervical lesions. Vaccine. 2019;37:4262-7.

68. Bosch FX, Robles C, Diaz M, Arbyn M, Baussano I, Clavel C, et al. HPV-FASTER: broadening the scope for prevention of HPV-related cancer. Nat Rev Clin Oncol. 2016;13:119-32.

69. León-Maldonado L, Cabral A, Brown B, Ryan GW, Maldonado A, Salmerón J, et al. Feasibility of a combined strategy of HPV vaccination and screening in Mexico: the FASTER-Tlalpan study experience. Hum Vaccin Immunother. 2019;15:198694.

70. Janitzek CM, Peabody J, Thrane S, Carlsen P, Theander T, Salanti A, et al. A proofof-concept study for the design of a VLP-based combinatorial HPV and placental malaria vaccine. Sci Rep. 2019;9:5260.

71. Gohar A, Abdeltawab NF, Shehata N, Amin MA. Preclinical study of safety and immunogenicity of combined rubella and human papillomavirus vaccines: towards enhancing vaccination uptake rates in developing countries. Papillomavirus Res. 2019;8:100172.

72. Miao Y, Mzolo T, Pellegrini M. Immunogenicity of a quadrivalent human papillomavirus vaccine when co-administered with tetanus-reduced diphtheria-acellular pertussis and quadrivalent meningococcal conjugate vaccines in healthy adolescents: results from a randomized, observer-blind, controlled trial. Infect Dis Ther. 2019;8:335-41.

73. de Sanjosé S, Serrano B, Tous S, Alejo M, Lloveras B, Quirós B, et al. Burden of human papillomavirus (HPV)-related cancers attributable to HPVs 6/11/16/18/ 31/33/45/52 and 58. JNCI Cancer Spectr. 2019;2: pkyo45.

74. Hall MT, Simms KT, Lew JB, Smith MA, Brotherton JM, Saville M, et al. The projected timeframe until cervical cancer elimination in Australia: a modelling study. Lancet Public Health. 2019;4:e19-e27.

75. Simms KT, Steinberg J, Caruana M, Smith MA, Lew JB, Soerjomataram I, et al. Impact of scaled up human papillomavirus vaccination and cervical screening and the potential for global elimination of cervical cancer in 181 countries, 2020-99: a modelling study. Lancet Oncol. 2019;20:394-407. 